\title{
Association of GEMIN4 gene polymorphism and the risk of cancer: a meta-analysis
}

This article was published in the following Dove Press journal:

OncoTargets and Therapy

2 November 2017

Number of times this article has been viewed

\author{
Nan Wu \\ Xiaowei Zhang \\ Jinlong Tian \\ Shuang Yu \\ Ying Qiao
}

The Core Laboratory for Public Health Science and Practice, The First Affiliated Hospital of China Medical University, Shenyang, People's Republic of China
Correspondence: Ying Qiao

The Core Laboratory for Public Health Science and Practice, The First Affiliated Hospital of China Medical University, I55th North of Nanjing Street, Heping District, Shenyang I I000I, Liaoning,

People's Republic of China

Tel/fax +86248328 2728

Email qfm1932@aliyun.com

\begin{abstract}
Gem-associated protein 4 (GEMIN4) gene is a key regulator for the miRNA biogenesis processes. Recent studies have demonstrated that some single-nucleotide polymorphisms (SNPs) in GEMIN4 gene are associated with the risk of cancer, but the results are still controversial. Therefore, we conducted a meta-analysis to analyze the association between three major SNPs (rs2740348, rs7813, and rs3744741) in the GEMIN4 gene and the risk of cancer. Relevant articles were searched in Web of Science, PubMed, Cochrane Library, Chinese Wan Fang, and Chinese National Knowledge Infrastructure databases. Pooled odds ratio (OR) with 95\% confidence interval (CI) was calculated to quantitatively estimate the association. Publication bias and sensitivity analyses were undertaken to evaluate the stability of the results. Overall, the pooled results showed that rs 2740348 involving 3,604 cases and 3,770 controls was significantly associated with increased cancer risk ( $\mathrm{GG}$ vs $\mathrm{GC} / \mathrm{CC}$ : $\mathrm{OR}=1.16,95 \% \mathrm{CI}=1.05-1.29, P=0.004)$ and rs7813 involving 4,729 cases and 4,562 controls was also related to increased cancer risk (TT vs TC/CC: $\mathrm{OR}=1.12,95 \% \mathrm{CI}=1.03-1.22, P=0.009$ ). However, there was no significant association between rs3744741 and cancer risk under overall genetic models. In conclusion, our study has demonstrated that rs2740348 and rs7813 are associated with increased risk of cancer, and they may be new biomarkers for predicting cancer risk.
\end{abstract}

Keywords: gem-associated protein 4, single-nucleotide polymorphism, cancer, meta-analysis

\section{Introduction}

Cancer is a major serious challenge to human health worldwide. According to the latest report from American Cancer Society, 1,688,780 new cancer cases and 600,920 cancer deaths are expected to occur in the United States in $2017 .{ }^{1}$ Cancer is considered as a multi-genetic disease caused by substantial accumulation of DNA mutations. ${ }^{2}$ Genetic variations will induce the activation of oncogenes and inactivation of tumor suppressor genes, subsequently resulting in unrestrained cell proliferation and neoplasm formation. ${ }^{3,4}$ With cancer genome sequencing widely applied, identifying the mutations in an individual patient may contribute to preventing cancer efficiently. ${ }^{5}$

MicroRNA (miRNA), a type of noncoding RNA containing approximately 22 nucleotides, participates in regulating gene expression at the post-transcriptional level. ${ }^{6}$ miRNA biogenesis is a sophisticated process involving several steps. To be specific, miRNA is initially transcribed into a 300- to 1,000-nucleotide-length pri-miRNA, ${ }^{7}$ and then the pri-miRNA is processed into two-nucleotide strands precursor-miRNA (pre-miRNA) in the nucleus in collaboration with a nuclear protein known as DiGeorge Syndrome Critical Region 8 (DGCR8). ${ }^{8}$ Finally, the pre-miRNA is further cut by the RNase III enzyme Dicer in the cytoplasm to generate a mature miRNA. ${ }^{9}$ Gem-associated protein 4 (GEMIN4), encoded by GEMIN4 gene, along with the mature miRNA, Argonaute (Ago) protein and gem-associated protein 3 (GEMIN3) 
participates in the formation of the miRNA-induced silencing complex (RISC), which promotes the mature miRNAs to bind to their target mRNAs and then regulates the expression of target genes. ${ }^{10,11}$

Recent studies have found that miRNAs are generally dysregulated in cancer and function as oncogenes or tumor suppressor genes. ${ }^{12,13}$ Furthermore, the alteration in miRNA biogenesis genes may be associated with susceptibility to cancer probably by influencing the function of miRNAs. ${ }^{14}$ Furthermore, a body of evidence has indicated that some single-nucleotide polymorphisms (SNPs) in the GEMIN4 gene, a major miRNA biogenesis gene, are associated with the risk of several types of cancer, ${ }^{15-19}$ but the published results are inconsistent. ${ }^{15-17}$ Therefore, in the present study, we undertook a meta-analysis to analyze the association between three major SNPs in the GEMIN4 gene (rs2740348, rs7813, and rs3744741) and the risk of cancer based on published articles.

\section{Methods}

\section{Literature search}

Relevant articles published prior to July 20, 2017 were mainly identified through searches of Web of Science, PubMed, Cochrane Library, Chinese Wan Fang, and Chinese National Knowledge Infrastructure databases online. The key terms of literature search included "genetic polymorphism," "single nucleotide polymorphism," "genetic variants," "nuclear organelle associated protein 4," "GEMIN4," "cancer," "carcinoma," "malignancy," and "tumor." Furthermore, manual retrieval was undertaken additionally by browsing the references from eligible articles. Each retrieved article including title, abstract, and full text was comprehensively evaluated by two independent authors to determine whether it could be included in the final analysis. If their opinions were not consistent, other authors critically assessed whether the retrieved article should be included.

\section{Eligibility criteria}

The qualified articles should simultaneously meet three major inclusion criteria as follow: 1) concerning the association between the GEMIN4 polymorphism and cancer; 2) casecontrol study; and 3) complete data concerning the frequencies of genotype or sufficient original data for calculating the frequencies of genotype. Retrieved articles were excluded if they met one of the two major exclusion criteria as follows: 1) duplicated or retracted articles, 2) review, case report, letter, or short commentary. In addition, the healthy control should be free of any type of cancer.

\section{Data extraction}

Information about the eligible article including the author's name, publication date, characteristics of case and control, methods of genotyping, $P$-value of the Hardy-Weinberg equilibrium (HWE) test, sample size of the study, and number of genotype was extracted from full text by two independent authors separately. The Newcastle-Ottawa Scale (NOS) was used to evaluate the quality of included studies as described previously. ${ }^{20}$

\section{Statistical analysis}

Firstly, the distribution of genotype in the control was evaluated to ascertain whether it conformed to the HWE through the chi-square goodness-of-fit test. Pooled odds ratio (OR) with $95 \%$ confidence interval (CI) was calculated to estimate the association between the GEMIN4 polymorphism and risk of cancer. Cochran's Q statistic in combination with the I-squared $\left(I^{2}\right)$ metric was used to assess heterogeneity. Specifically, $P<0.10$ or $I^{2}>50 \%$ was considered as indicative of significant heterogeneity. The random-effects model was used to estimate the pooled OR with significant heterogeneity existing; otherwise, the fixed-effects model was developed. The best-fitting genetic model was chosen to estimate the association between the GEMIN4 polymorphism and the risk of cancer, according to previously published articles. ${ }^{21}$ Subgroup analysis was further undertaken based on ethnicity. Publication bias was evaluated through funnel plots in combination with Egger's linear regression test. Publication bias was considered significant when the $P$-value of Egger's test was less than 0.05 . Sensitivity analysis was conducted to evaluate the stability of pooled results through omitting a single study each time to examine its influence on the total OR value. Statistical data analyses were conducted by using STATA 11.0 (STATA Corp., College Station, TX, USA).

\section{Results}

\section{Characteristics of the included studies}

In general, 70 relevant articles were originally chosen from different electronic databases. According to the inclusion and exclusion criteria, 55 articles were eliminated and 15 qualified articles ( 9 articles referring to rs2740348, ${ }^{15,18,22,28}$ 11 articles referring to rs 7813, ${ }^{15-17,19,22-25,29-31}$ and 5 articles referring to rs3744741 $15,16,22-24)$ were finally included in the meta-analysis (Figure 1). Specifically, details of the eliminated articles are shown in Table S1.

The main characteristics of all qualified studies are listed in Tables 1-3, respectively. Each included study got no less 


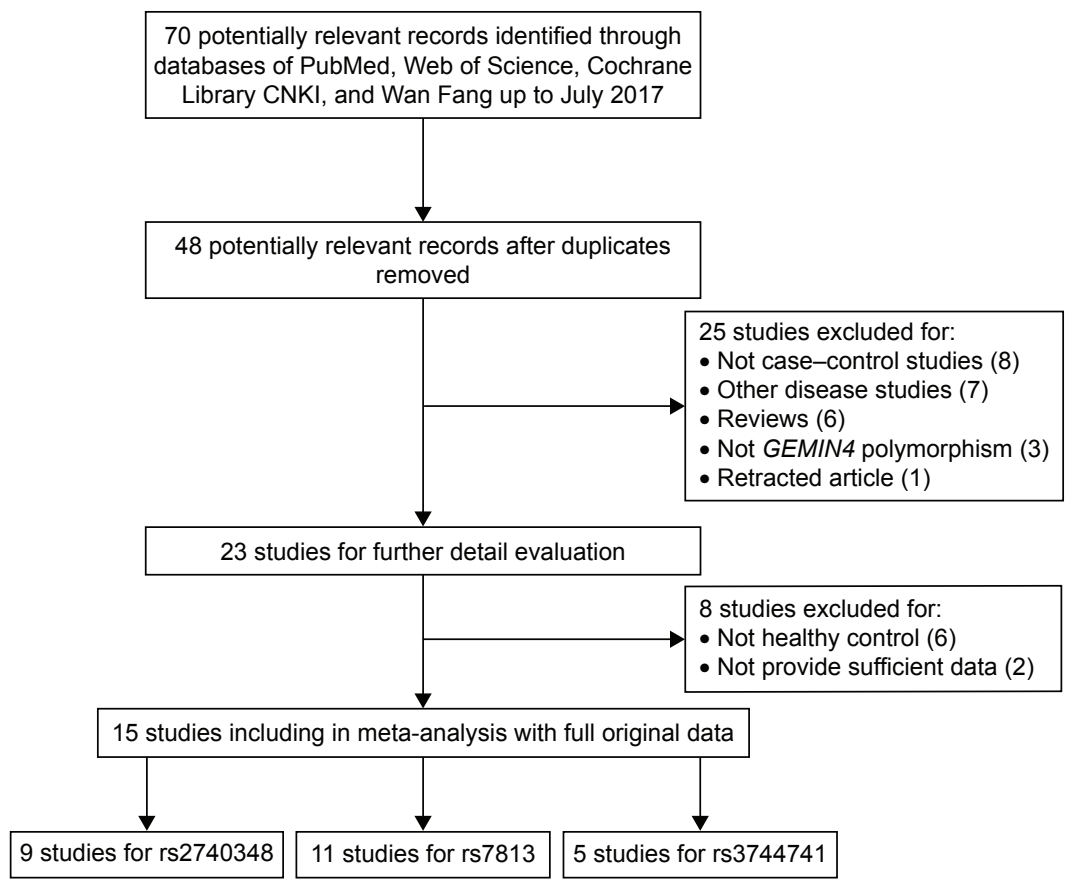

Figure I Flow chart of the study selection process.

than 7 points referring to the NOS score, and the genotype distribution in the control conformed to HWE.

\section{Quantitative data synthesis}

For rs2740348, the pooled result with 3,604 cases and 3,770 controls showed that it was significantly associated with increased risk of cancer in the recessive model (GG vs $\mathrm{GC} / \mathrm{CC}: \mathrm{OR}=1.16,95 \% \mathrm{CI}=1.05-1.29, P=0.004$; Figure $2 \mathrm{~A}$ ). The result of subgroup analysis by ethnicity showed that rs2740348 was significantly associated with increased risk of cancer in a mixed population (GG vs GC/CC: $\mathrm{OR}=1.23$, 95\% CI $=1.05-1.43, P=0.009$; Figure $2 \mathrm{~A}$ ), but not in Caucasians (GG vs GC/CC: $\mathrm{OR}=1.20,95 \% \mathrm{CI}=0.95-1.52$, $P=0.127$; Figure 2A) and Asians (GG vs GC/CC: $\mathrm{OR}=1.07$, 95\% CI $=0.90-1.27, P=0.423$; Figure $2 \mathrm{~A}$ ).

For rs7813, the pooled result with 4,729 cases and 4,562 controls also indicated that rs7813 was related to increased risk of cancer in the recessive model (TT vs TC/CC: $\mathrm{OR}=1.12,95 \% \mathrm{CI}=1.03-1.22, P=0.009$; Figure 2B). Subgroup analysis by ethnicity further indicated that rs7813 significantly increased susceptibility to cancer in Asians (TT vs TC/CC: $\mathrm{OR}=1.14,95 \% \mathrm{CI}=1.02-1.27, P=0.019$; Figure 2B) rather than Caucasians (TT vs TC/CC: $\mathrm{OR}=1.09$, $95 \% \mathrm{CI}=0.95-1.25, P=0.206$; Figure $2 \mathrm{~B}$ ).

As for rs3744741, the pooled result with 2,167 cases and 2,143 controls suggested no association existed between rs 3744741 and the risk of cancer in the recessive model
(CC vs CT/TT: $\mathrm{OR}=1.04,95 \% \mathrm{CI}=0.92-1.18, P=0.509$; Figure 2C). Furthermore, no association was found in other genetic models (Table S2).

\section{Sensitivity analysis and publication bias}

To evaluate the stability of the pooled results, sensitivity analysis was conducted. The result of sensitivity analysis showed the pooled OR value did not significantly change when omitting any single study (Figure 3 ). To evaluate the publication bias, the funnel plot and Egger's linear regression test were applied. Funnel plots of rs2740348, rs7813, and rs3744741 were visually symmetrical (Figure 4) and, furthermore, the result of Egger's test showed no evidence of publication bias ( $P=0.769,95 \%$ CI: $-2.90,2.24$ for rs 2740348 , $P=0.751,95 \% \mathrm{CI}:-3.61,4.83$ for $\operatorname{rs} 7813, P=0.557$, 95\% CI: $-4.03,6.14$ for $\mathrm{rs} 3744741$, respectively).

\section{Discussion}

GEMIN4 gene is located on the human chr17. Genetic variants in the GEMIN4 gene might lead to a decreased inhibitory effect on the proliferation of hepatocarcinoma cells and simultaneously have an impact on the DNA repair system by altering the expression of proliferation-associated genes and DNA repair genes. ${ }^{32}$ Several studies have found that genetic variants in the GEMIN4 gene not only alter the susceptibility to cancer, ${ }^{15-19}$ but also influence the survival time of patients with cancer. ${ }^{33,34}$ However, as far as we know, 

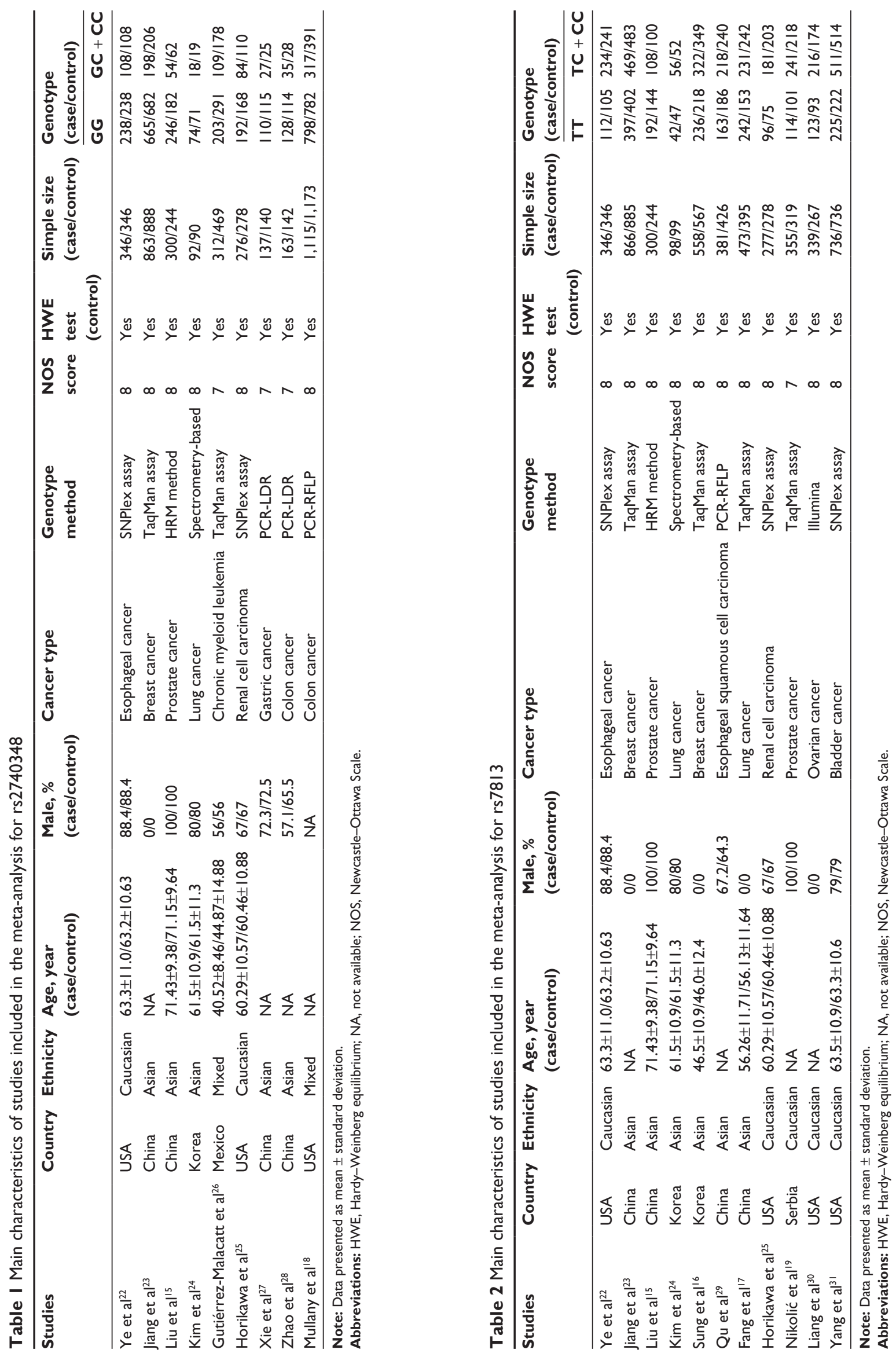


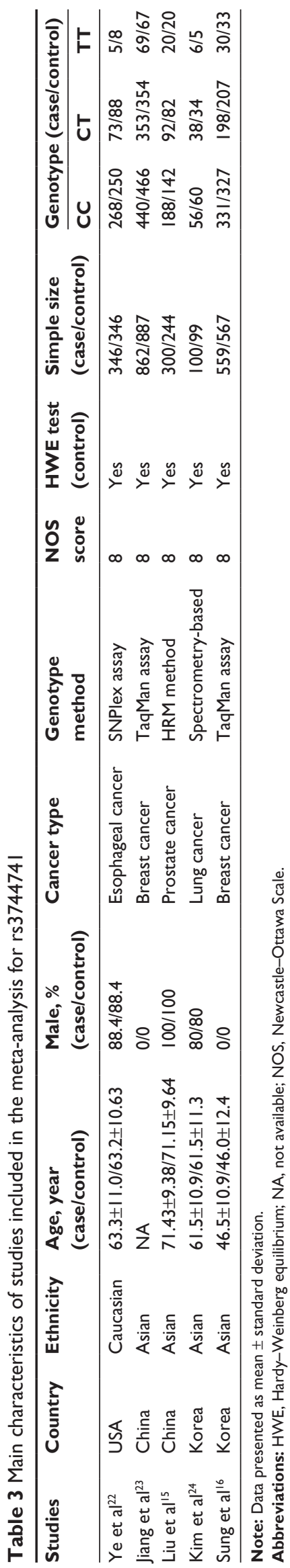

most of these studies are small-scale case-control studies. Although a meta-analysis conducted by Zhu et al firstly showed that rs2740348 and rs7813 polymorphisms were associated with increased risk of cancer, the total number of subjects in their meta-analysis was also limited. ${ }^{35}$ Compared with previous meta-analysis, one advantage of our metaanalysis is that it included larger sample size, nearly double that of studies earlier. In addition, another new finding of our study is that it firstly evaluates the association between the rs3744741 polymorphism and the risk of cancer.

The rs2740348 polymorphism is a $\mathrm{C} / \mathrm{G}$ mutation located in the exon region of the GEMIN4 gene. Liu et al found that rs2740348 was associated with decreased risk of prostate cancer. ${ }^{15} \mathrm{~A}$ similar protective effect was also shown in colon cancer. ${ }^{18}$ However, Zhao et al suggested no association existed in colorectal cancer. ${ }^{28}$ Moreover, there was no association with lung cancer, ${ }^{24}$ chronic myeloid leukemia, and gastric cancer. ${ }^{26,27}$ More interestingly, Yang et al found that a haplotype of the GEMIN4 gene including rs2740348 was associated with increased risk of bladder cancer. ${ }^{31}$ In our present meta-analysis, we found that rs 2740348 was significantly associated with increased risk of cancer in the general population under the recessive model ( $\mathrm{GG}$ vs $\mathrm{GC} / \mathrm{CC}$ ), which agreed with the result reported by Zhu et al. ${ }^{35}$ However, this conclusion was only confirmed in a mixed population, but not in Asians and Caucasians. Therefore, the discrepancy of the results might be partly attributed to the difference of the study population.

The rs7813 polymorphism is a $\mathrm{C} / \mathrm{T}$ mutation located in the exon region of the GEMIN4 gene. Liu et al found that rs7813 was associated with an increased risk of prostate cancer, ${ }^{15}$ and Nikolić et al also found a significant association between rs7813 and the risk of prostate cancer progression. ${ }^{19}$ However, Fang et al found that rs7813 was associated with decreased risk of lung cancer. ${ }^{17}$ A similar protective effect was found in ovarian and breast cancers. ${ }^{30,34}$ In addition, some studies did not report any significant association between rs7813 and the risk of breast cancer, ${ }^{23}$ lung cancer, and esophageal squamous cell carcinoma. ${ }^{24,32}$ In the present study, we reported that rs 7813 was related to an increased risk of cancer in the general population under the recessive model (TT vs TC/CC), but this conclusion was only confirmed in Asians, not in Caucasians, which is contrary to a previous meta-analysis. ${ }^{35}$ Thus, we inferred that the discrepancy might be explained by the diversity of study population and tumor types.

The rs 3744741 polymorphism is a $\mathrm{C} / \mathrm{T}$ mutation located in the exon region of the GEMIN4 gene. Liu et al found that 


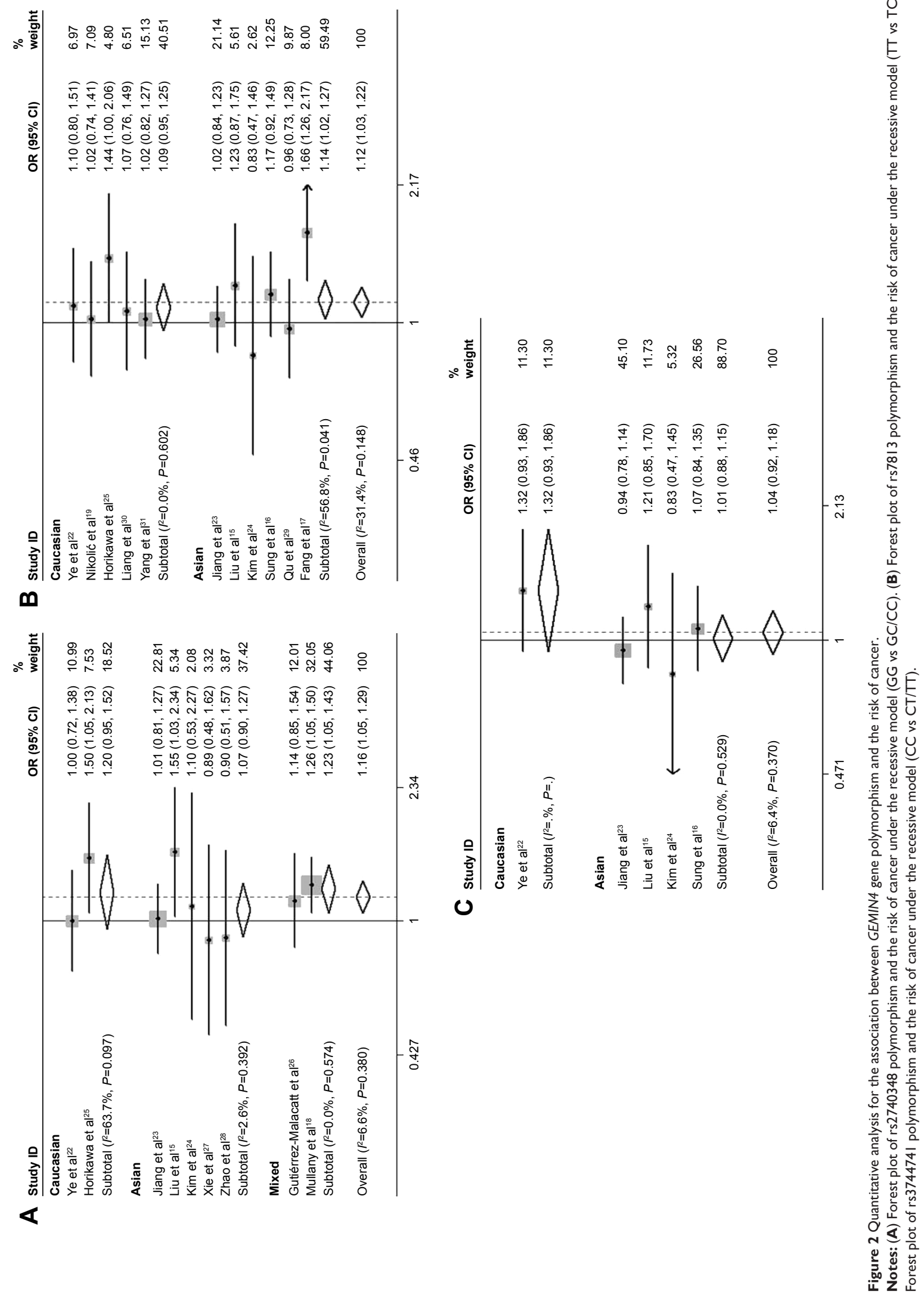




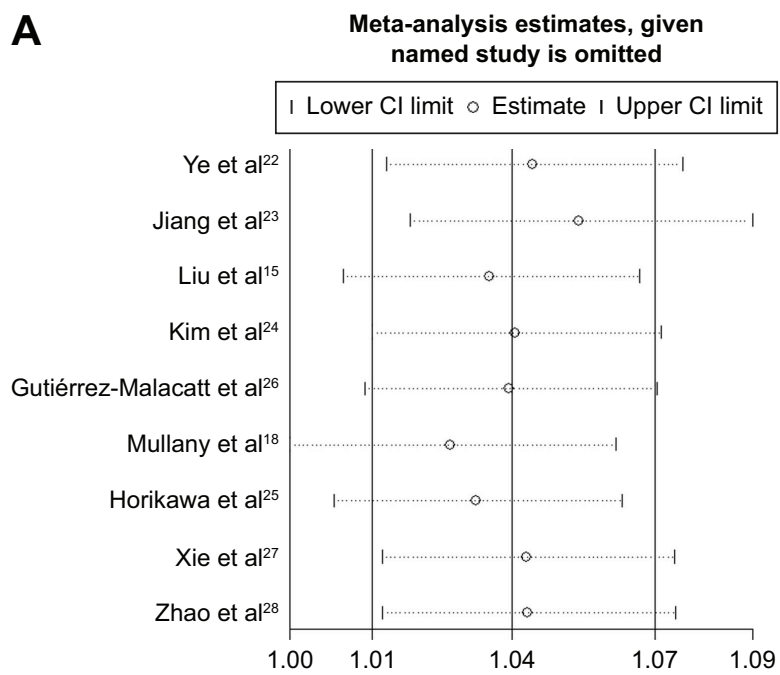

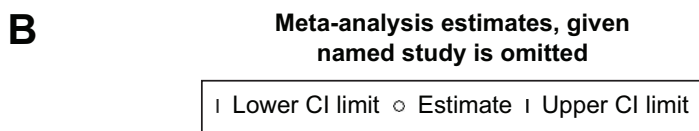

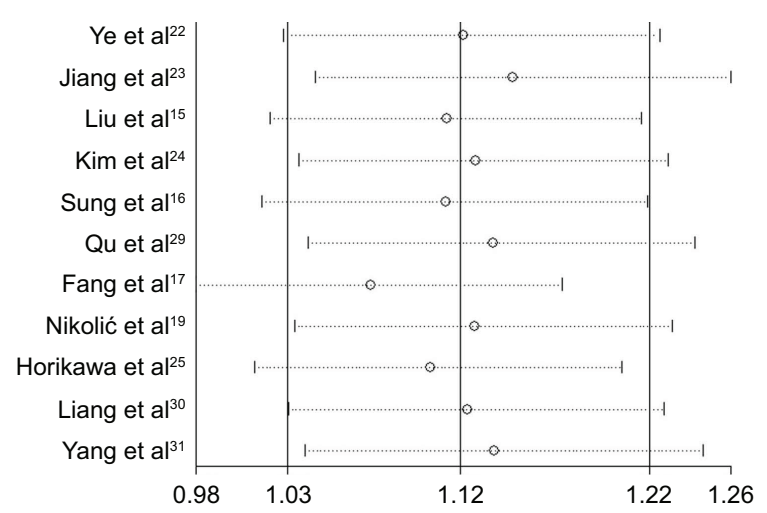

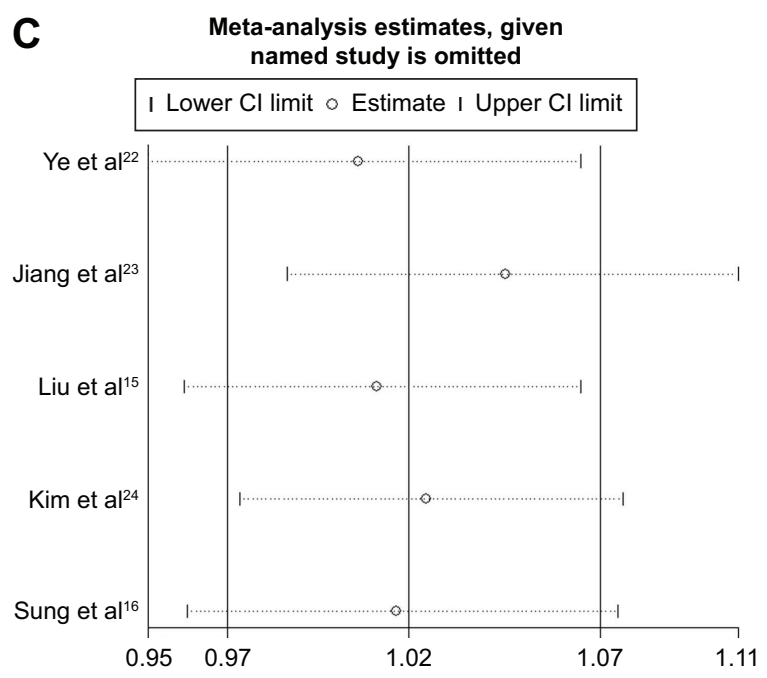

Figure 3 Sensitivity analysis for the association of GEMIN4 gene polymorphism with the risk of cancer.

Notes: (A) Sensitivity analysis for rs 2740348 polymorphism and the risk of cancer under the recessive model (GG vs GC/CC). (B) Sensitivity analysis for rs78। 3 polymorphism and the risk of cancer under the recessive model (TT vs TC/CC). (C) Sensitivity analysis for rs374474I polymorphism and the risk of cancer under the recessive model (CC vs CT/TT).

the $\mathrm{T}$ allele of rs3744741 was associated with decreased risk of III/IV stage of prostate cancer, ${ }^{15}$ but no significant association between rs7813 and the risk of cancer was reported in breast and lung cancers. ${ }^{23,24}$ Moreover, we did not find any significant associations between rs7813 and the risk of cancer, neither in Asians nor in Caucasians under overall genetic models. Considering that the sample size was relatively small (2,167 cases and 2,143 controls), this conclusion should be further validated by some more large-scale studies.

Certainly, several limitations still existed in our metaanalysis. Firstly, the sample size of our meta-analysis, especially for rs3744741, was still relatively small. The conclusion should be reconfirmed by more large-scale studies. Secondly, the characteristics of included studies, such as age and gender, were diverse and might affect the results of meta-analysis Due to a lack of valid data pertaining to age and gender for all included studies, we could not evaluate their impacts on the pooled OR value. Finally, our study only evaluated the impact of these three SNPs on the risk of cancer separately and ignored the interaction effects of multiple SNPs, which was a deficit we must acknowledge.

\section{Conclusion}

Our study has demonstrated that rs2740348 and rs7813 polymorphism in GEMIN4 gene are risk factors for cancer, but rs3744741 polymorphism is not associated with the risk of cancer. These findings should be further reconfirmed by more large-scale studies. 

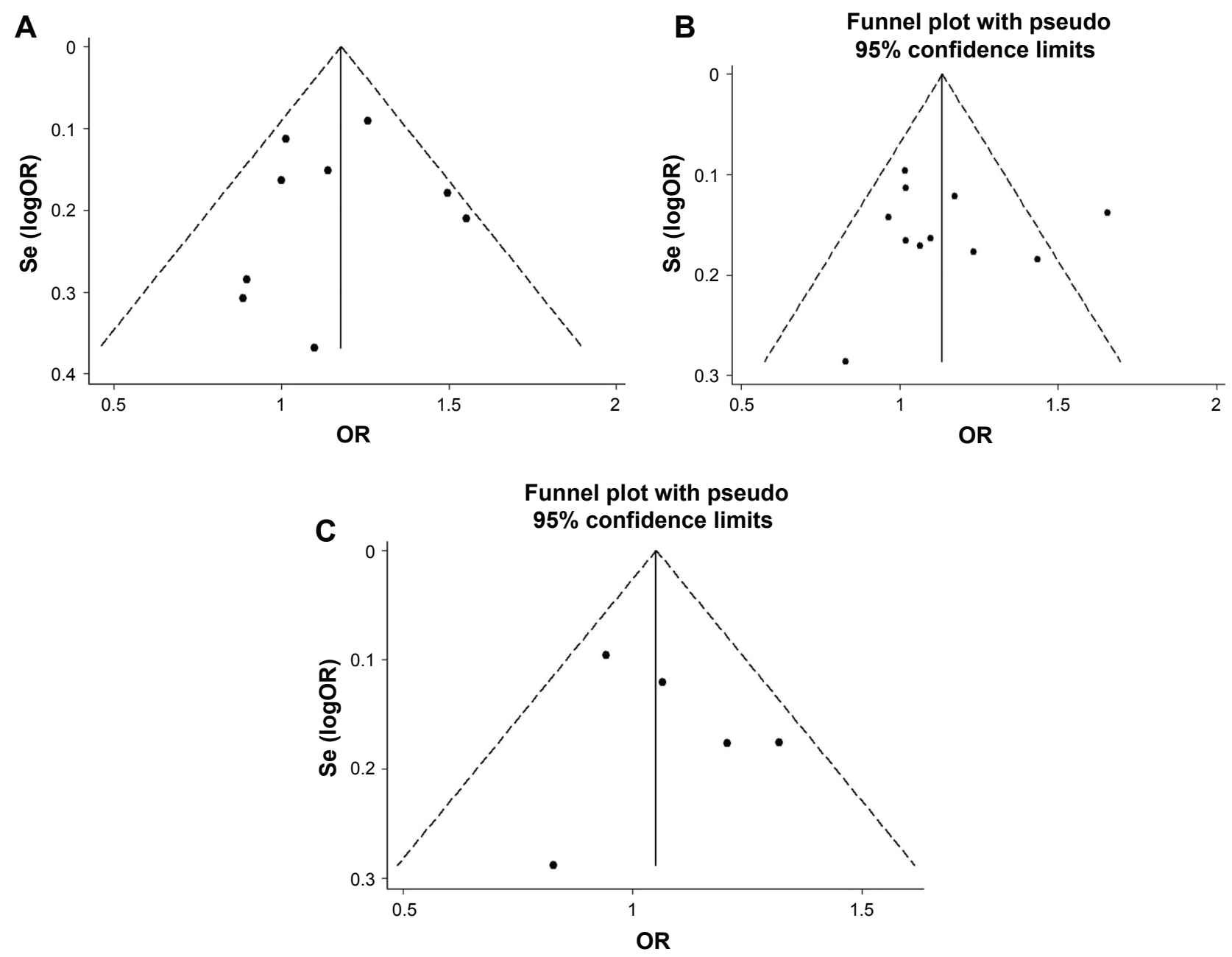

Figure 4 Funnel plot of GEMIN4 gene polymorphism and the risk of cancer.

Notes: (A) Funnel plot of rs2740348 polymorphism and the risk of cancer under the recessive model (GG vs GC/CC). (B) Funnel plot of rs78I3 polymorphism and the risk of cancer under the recessive model (TT vs TC/CC). (C) Funnel plot of rs374474I polymorphism and the risk of cancer under the recessive model (CC vs CT/TT).

\section{Disclosure}

The authors report no conflicts of interest in this work.

\section{References}

1. Siegel RL, Miller KD, Jemal A. Cancer Statistics, 2017. CA Cancer J Clin. 2017;67(1):7-30.

2. Novak K. Epigenetics changes in cancer cells. Med Gen Med. 2004; 6(4): 17 .

3. Solomon H, Brosh R, Buganim Y, Rotter V. Inactivation of the p53 tumor suppressor gene and activation of the Ras oncogene: cooperative events in tumorigenesis. Discov Med. 2010;9(48):448-454.

4. Lichtenstein AV, Kisseljova NP. DNA methylation and carcinogenesis. Biochemistry (Mosc). 2001;66(3):235-255

5. Brennan P, Wild CP. Genomics of cancer and a new era for cancer prevention. PLoS Genet. 2015;11(11):e1005522.

6. Ambros V. The functions of animal microRNAs. Nature. 2004;431(7006): 350-355.

7. Lee Y, Kim M, Han J, et al. MicroRNA genes are transcribed by RNA polymerase II. EMBO J. 2004;23(20):4051-4060.

8. Yeom KH, Lee Y, Han J, Suh MR, Kim VN. Characterization of DGCR8/ Pasha, the essential cofactor for Drosha in primary miRNA processing. Nucleic Acids Res. 2006;34(16):4622-4629.

9. Lee Y, Ahn C, Han J, et al. The nuclear RNase III Drosha initiates microRNA processing. Nature. 2003;425(6956):415-419.
10. Sontheimer EJ. Assembly and function of RNA silencing complexes. Nat Rev Mol Cell Biol. 2005;6(2):127-138.

11. Bartel DP. MicroRNAs: genomics, biogenesis, mechanism, and function. Cell. 2004;116(2):281-297.

12. Hayes J, Peruzzi PP, Lawler S. MicroRNAs in cancer: biomarkers, functions and therapy. Trends Mol Med. 2014;20(8):460-469.

13. Wang W, Luo YP. MicroRNAs in breast cancer: oncogene and tumor suppressors with clinical potential. J Zhejiang Univ Sci B. 2015;16(1): $18-31$.

14. Gregory RI, Shiekhattar R. MicroRNA biogenesis and cancer. Cancer Res. 2005;65(9):3509-3512.

15. Liu J, Liu J, Wei M, et al. Genetic variants in the microRNA machinery gene GEMIN4 are associated with risk of prostate cancer: a case-control study of the Chinese Han population. DNA Cell Biol. 2012;31(7): 1296-1302.

16. Sung H, Lee KM, Choi JY, et al. Common genetic polymorphisms of microRNA biogenesis pathway genes and risk of breast cancer: a casecontrol study in Korea. Breast Cancer Res Treat. 2011;130(3):939-951.

17. Fang X, Yin Z, Li X, Xia L, Zhou B. Polymorphisms in GEMIN4 and AGO1 genes are associated with the risk of lung cancer: a case-control study in Chinese female non-smokers. Int J Environ Res Public Health. 2016;13(10). pii: E939.

18. Mullany LE, Herrick JS, Wolff RK, Buas MF, Slattery ML. Impact of polymorphisms in microRNA biogenesis genes on colon cancer risk and microRNA expression levels: a population-based, case-control study. BMC Med Genomics. 2016;9(1):21. 
19. Nikolić Z, Savić Pavićević D, Vučić N, Cerović S, Vukotić V, Brajušković G. Genetic variants in RNA-induced silencing complex genes and prostate cancer. World J Urol. 2017;35(4):613-624.

20. Stang A. Critical evaluation of the Newcastle-Ottawa scale for the assessment of the quality of nonrandomized studies in meta-analyses. Eur J Epidemiol. 2010;25(9):603-605.

21. Lin J, Horikawa Y, Tamboli P, Clague J, Wood CG, Wu X. Genetic variations in microRNA-related genes are associated with survival and recurrence in patients with renal cell carcinoma. Carcinogenesis. 2010;31(10):1805-1812.

22. Ye Y, Wang KK, Gu J, et al. Genetic variations in microRNA-related genes are novel susceptibility loci for esophageal cancer risk. Cancer Prev Res (Phila). 2008;1(6):460-469.

23. Jiang Y, Chen J, Wu J, et al. Evaluation of genetic variants in microRNA biosynthesis genes and risk of breast cancer in Chinese women. Int $J$ Cancer. 2013;133(9):2216-2224.

24. Kim JS, Choi YY, Jin G, et al. Association of a common AGO1 variant with lung cancer risk: a two-stage case-control study. Mol Carcinog. 2010;49(10):913-921.

25. Horikawa Y, Wood CG, Yang H, et al. Single nucleotide polymorphisms of microRNA machinery genes modify the risk of renal cell carcinoma. Clin Cancer Res. 2008;14(23):7956-7962.

26. Gutiérrez-Malacatt H, Ayala-Sanchez M, Aquino-Ortega X, et al. The rs61764370 functional variant in the KRAS oncogene is associated with chronic myeloid leukemia risk in women. Asian Pac J Cancer Prev. 2016;17(4):2265-2270.

27. Xie Y, Wang Y, Zhao Y, Guo Z. Single-nucleotide polymorphisms of microRNA processing machinery genes are associated with risk for gastric cancer. Onco Targets Ther. 2015;8:567-571.
28. Zhao Y, Du Y, Zhao S, Guo Z. Single-nucleotide polymorphisms of microRNA processing machinery genes and risk of colorectal cancer. Onco Targets Ther. 2015;8:421-425.

29. Qu Y, Qu H, Luo M, et al. MicroRNAs related polymorphisms and genetic susceptibility to esophageal squamous cell carcinoma. Mol Genet Genomics. 2014;289(6):1123-1130.

30. Liang D, Meyer L, Chang DW, et al. Genetic variants in MicroRNA biosynthesis pathways and binding sites modify ovarian cancer risk, survival, and treatment response. Cancer Res. 2010;70(23):9765-9776.

31. Yang H, Dinney CP, Ye Y, Zhu Y, Grossman HB, Wu X. Evaluation of genetic variants in microRNA-related genes and risk of bladder cancer. Cancer Res. 2008;68(7):2530-2537.

32. Wan D, He M, Wang J, et al. Two variants of the human hepatocellular carcinoma-associated HCAP1 gene and their effect on the growth of the human liver cancer cell line Hep3B. Genes Chromosomes Cancer. 2004;39(1):48-58.

33. Yang PW, Huang YC, Hsieh CY, et al. Association of miRNA-related genetic polymorphisms and prognosis in patients with esophageal squamous cell carcinoma. Ann Surg Oncol. 2014;21(Suppl 4): S601-S609.

34. Sung $\mathrm{H}$, Jeon $\mathrm{S}$, Lee KM, et al. Common genetic polymorphisms of microRNA biogenesis pathway genes and breast cancer survival. $B M C$ Cancer. 2012;12:195.

35. Zhu W, Zhao J, He J, et al. Genetic variants in the MicroRNA biosynthetic pathway Gemin 3 and Gemin 4 are associated with a risk of cancer: a meta-analysis. Peer J. 2016;4:e1724.
OncoTargets and Therapy

\section{Publish your work in this journal}

OncoTargets and Therapy is an international, peer-reviewed, open access journal focusing on the pathological basis of all cancers, potential targets for therapy and treatment protocols employed to improve the management of cancer patients. The journal also focuses on the impact of management programs and new therapeutic agents and protocols on

\section{Dovepress}

patient perspectives such as quality of life, adherence and satisfaction. The manuscript management system is completely online and includes a very quick and fair peer-review system, which is all easy to use. Visit http://www.dovepress.com/testimonials.php to read real quotes from published authors. 